\title{
NUMERICAL MODEL OF THE MULTIPHASE VORTEX FLOW IN THE PRESENCE OF A POWERFUL VOLUMETRIC HEAT SOURCE
}

\section{Marek Mlkvik, Peter Hlbočan, Branislav Knížat}

Strojnícka Fakulta STU v Bratislave, Nám. Slobody 17, 812 31, Bratislava, marek.mlkvik@stuba.sk, peter.hlbocan@stuba.sk, branislav.knizat@stuba.sk

Keywords: multiphase flow, plasma flow, CFD

\begin{abstract}
The presented paper focuses on the CFD analysis of flows with a dominant tangential velocity component. A strong volumetric heat source is also present in the computational domain. This heat source represents the electric arc which heats the working medium and produces the plasma. The influence of the boundary conditions used herein on the presented flow is then discussed.
\end{abstract}

\section{INTRODUCTION}

Many technological processes use ionized, highly heated gas (plasma) in their working cycle. The device used to generate plasma is called a plasmatron. Plasma in these devices is generated by an electric arc. As a result of high electric current values in the electric arc, the temperature of the gas becomes increasing stronger. As an effect of the high temperatures, ionization of the gas is achieved, so plasma is then generated. This article deals with the plasmatron where the working medium is water at atmospheric pressure. The water is injected into the plasmatron by the tangential inlet channel, so the flow is characterized by high values of the tangential velocity component. Due to the presence of this powerful volumetric energy source (heat source represents the electric arc), the water evaporates. Water vapor then heats up due to the energy source and high temperatures and the process of ionization begins. Depending on the flowrate of the water at the inlet a thin water film on the walls of the plasmatron remains.

\section{MATHEMATICAL DESCRIPTION OF THEL PLASMA FLOW}

Internal volume of the described plasmatron has a conical shape with inlet channels in the tangential direction, used for injection of the working fluid (Figure 1). The flow in this domain can be described as compressible, non-isothermal flow of electrically conductive fluid with multiple phase changes. The mathematical description of such flows is represented by a set of differential equations.

The momentum equations for the transient flow of a viscous fluid can be written as (1). This equation represents the equilibrium of the volumetric and surface forces.

$$
\frac{\partial(\rho \cdot \vec{u})}{\partial t}+\nabla \cdot(\rho \cdot \vec{u} \cdot \vec{u})=-\nabla \cdot p+\nabla \cdot \tau_{i j}+\vec{F}_{L}
$$




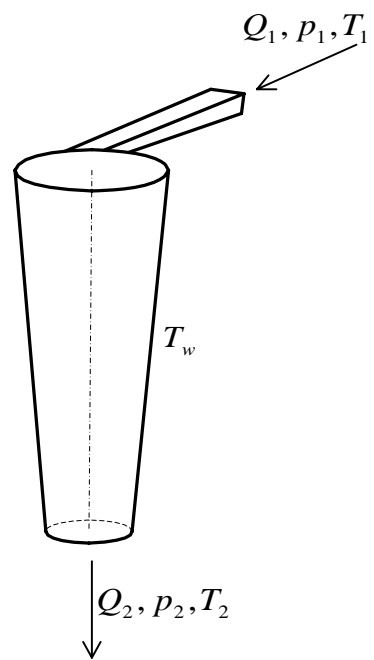

Figure 1: Internal volume of the plasmatron.

The mass conservation equation:

$$
\frac{\partial \rho}{\partial t}+\nabla \cdot(\rho \cdot \vec{u})=0
$$

The energy equation represents the equilibrium of the total change of the internal energy, heat flow, and the work of the described control volume (3).

$$
\frac{\partial e}{\partial t}+\nabla[(e+p) \cdot \vec{u}-\lambda \cdot \nabla \cdot T]=\vec{j} \cdot \vec{E}+\Phi_{\text {diss }}-\dot{R}
$$

In the case of plasma flow, the working fluid (ionized gas) is electrically conductive. This fact produces additional terms in the presented equations. For the momentum equation, the additional term is called the Lorentz force (4). This term represents the volumetric force due to the movement of the conductive fluid in the magnetic field.

$$
\vec{F}_{L}=\vec{j} \times \vec{B}
$$

In the energy equation (3), the electric current in the arc generates heat (Joule Heating). This heating is represented by the source term (5) in the energy equation (3).

$$
Q_{J}=\vec{j} \cdot \vec{E}
$$


The high temperatures of the electric arc (to the $10^{4}$ Kelvin) causes energy lost by radiation. The radiation term must be thus included into the simulation. There are different ways for computation of the radiation term. One of the ways is to use the net emission coefficients [1], [2]. The radiation term can be then written as (6).

$$
\dot{R}=4 \cdot \pi \cdot \varepsilon_{N}
$$

\section{COMPUTATIONAL DOMAIN AND BOUNDARY CONDITIONS}

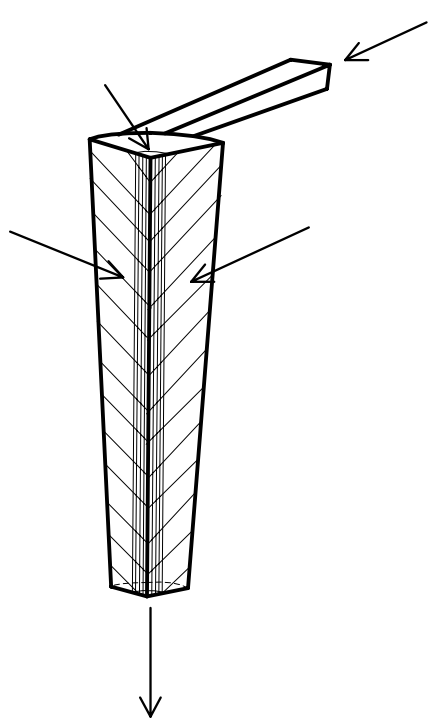

Figure 2: Sketch of the computation domain for CFD analysis of the plasma flow in the plasmatron

The rotational character of the internal volume of the plasmatron allows for the use of an Olumetric axisymetric computational domain (Figure 2). The grid elements are hexahedral while the whbeat source grid is structural, created in GAMBIT.

The boundary conditions and definition of the source term are set according to the expected parameters of the plasmatrons electric power source. An important aspect of the simulation is to find the regime, when most of the water, injected into the domain through inlet channels, become a vapor and then become a plasma due to the high temperatures. The inlet boundary condition is mass flow through the inlet channel. The used fluid is paer proplic temperature of $50 \mathrm{~K}$. The Outlet boundary condition is atmospheric pressure. The boundary 
condition at the walls is the wall temperature. This temperature can be set to a wide range of temperatures up to the maximum temperature which the material of the plasmatron can withstand.

The heat generated by the electric arc is currently included into the simulation as a volumetric heat source therm. The part of the domain, which generates the heat can be seen in Figure 2. The power of the volumetric heat source is set according to the expected working regimes of the plasmatron.

\section{MODELING OF THE TURBULENCE AND THE MULTIPHASE FLOW}

The flow in the described computational domain is very complicated and strongly affected by the conical shape of this domain. Because of the complexity of the flow, the correct turbulence model must be used. For the purposes of the described simulation, Reynolds Stress Model (RSM) gives the best results. This model is not dealing with the turbulent viscosity, but its computing the Reynolds stress tensor $\overline{\rho . u_{i}^{\prime} u_{j}^{\prime}}$ directly. The exact equation for the turbulent stress tensor (7) can be derived from Navier-Stokes equations.

$$
\begin{aligned}
& U_{k} \frac{\partial \overline{u_{i}^{\prime} u_{j}^{\prime}}}{x_{k}}=-\overline{u_{i}^{\prime} u_{k}^{\prime}} \cdot \frac{\partial U_{j}}{\partial x_{k}}-\overline{u_{j}^{\prime} u_{k}^{\prime}} \cdot \frac{\partial U_{i}}{\partial x_{k}}+\frac{P}{\rho}\left(\frac{\partial u_{i}^{\prime}}{\partial x_{j}}+\frac{\partial u_{j}^{\prime}}{\partial x_{i}}\right) \\
& -\frac{\partial}{\partial x_{k}} \cdot\left[\overline{u_{i}^{\prime} u_{j}^{\prime} u_{k}}+\frac{P u_{j}^{\prime}}{\rho} \cdot \delta_{i k}+\frac{P u_{i}}{\rho} \cdot \delta_{j k}-\frac{\mu}{\rho} \cdot \frac{\partial \overline{u_{i}^{\prime} u_{j}^{\prime}}}{\partial x_{k}}\right] \\
& -2 \cdot \frac{\mu}{\rho} \cdot \frac{\partial u_{i}}{x_{k}} \cdot \frac{\partial u_{j}^{\prime}}{x_{k}}
\end{aligned}
$$

Another aspect of the flow is multiple phase change of the working fluid. This fact makes the simulation more complex and complicated. From a physical point of view, there are three different phases, liquid water, water vapor, and ionized gas-plasma. Because the change of the physical properties during the ionization is continual, they can be modeled as one phase with physical properties including both, the vapor and the ionized gas. This approach is verified due to the very good correlation between experimental results [2], [3].

The phase change from liquid water to vapor can be computed by a simple mathematical model of the evaporation and condensation. The model used was based on the computation of the mass flow rate between different phases. This mass flow rate can be estimated by the equation (8).

$$
\dot{m}_{e \rightarrow v}=f \cdot \alpha_{1} \cdot p_{1} \cdot \frac{\left(T-T_{s a t}\right)}{T_{\text {sat }}}
$$




\section{RESULTS}

The simulation was focused on verifying the ability of the selected turbulence model to compute the correct vortex character of the flow. It was very important to retain the tangential velocity component of the flow in every part of the internal volume of the plasmatron. Another important attribute of the simulation was working with the multiphase flow. In this respect it was very important to retain a water film in the walls of the plasmatron, which is used for protecting and cooling the material of the plasmatron.

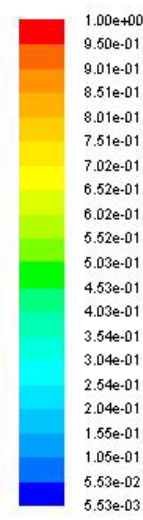

$$
\begin{aligned}
& 1.00 \mathrm{e}+00 \\
& 9.50 \mathrm{e}-01 \\
& 9.01 \mathrm{e}-01 \\
& 8.51 \mathrm{e}-01 \\
& 8.01 \mathrm{e}-01 \\
& 7.51 \mathrm{e}-01 \\
& 7.02 \mathrm{e}-01 \\
& 6.52 \mathrm{e}-01 \\
& 6.02 \mathrm{e}-01 \\
& 5.52 \mathrm{e}-01 \\
& 5.03 \mathrm{e}-01 \\
& 4.53 \mathrm{e}-01 \\
& 4.03 \mathrm{e}-01 \\
& 3.54 \mathrm{e}-01 \\
& 3.04 \mathrm{e}-01 \\
& 2.54 \mathrm{e}-01 \\
& 2.04 \mathrm{e}-01 \\
& 1.55 \mathrm{e}-01 \\
& 1.05 \mathrm{e}-01 \\
& 5.53 \mathrm{e}-02 \\
& 5.53 \mathrm{e}-03
\end{aligned}
$$

a) $2 g / s$

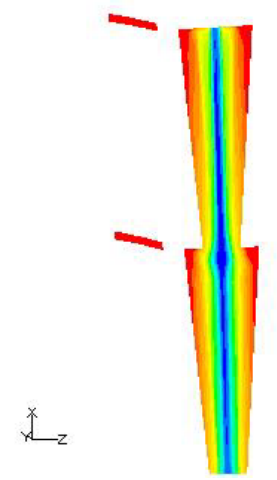

b) $8 \mathrm{~g} / \mathrm{s}$

Figure 3: Distribution of the water vapor fraction in the computational domain for different inlet mass flows

As can be seen in the Figure 3, the thickness of the water film was dependent on the selected mass flow of the water inlet (for the same heat generated by the electric arc). The inlet mass flow rate also effects the tangential velocity component of the flow. Distribution of the tangential velocity in the computational domain can be observed in the Figure 4. 


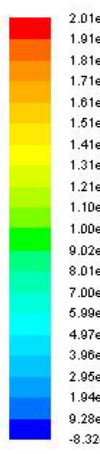

a) $2 g / s$

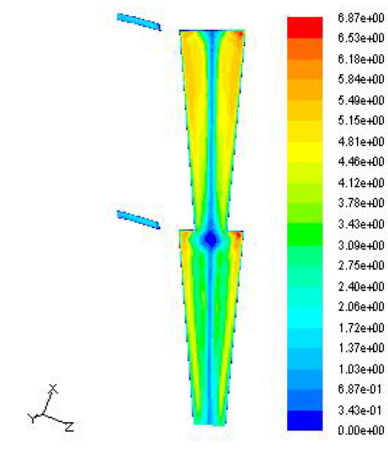

$\stackrel{x}{x}$

b) $8 g / s$

Figure 4: Distribution of the tangential velocity component in the computational domain for different inlet mass flows

\section{CONCLUSION}

A simplified numerical model of the plasma flow was introduced. The current version of the CFD model works with the non-isothermal flow with a phase change as well as with wide range of values for the physical properties at different temperatures. Neglected aspects of the flow were the volumetric forces affecting the flow due to electric conductivity of the plasma and the radiation losses due to high temperatures of the plasma. The next step in the development of the CFD model of the plasma flow is the inclusion of these aspects into the model. The comparison of the results from the advanced model will be published soon were the comparison with the experimental results will be discussed.

\section{Acknowledgement}

This publication is the result of the project implementation: "Applied research of technology of thermal plasma processes", ITMS code 26240220070, supported by the Research \& Development Operational Programme funded by the ERDF.

\section{REFERENCES}

[1] J. Jeništa, Numerical Modeling of Hybrid Stabilized Electric Arc with Uniform Mixing of Gases . IEEE Trans. on Plasma Sci. 32 (2), 464-472 Part 1, APR 2004

[2] J. Jeništa, Numerical Modeling of Hybrid Stabilized Electric Arc with Uniform Mixing of Gases . IEEE Trans. on Plasma Sci. 32 (2), 464-472 Part 1, APR 2004

[3] J. Jeništa, M. Bartlová, V. Aubrecht, Performance of Water and Hybrid Stabilized Electric Arcs: the Impact of Dependence of Radiation Losses and Plasma Density on Pressure . Czechoslovak Journal of Physics 56 (Suppl.B), B1224-B1230, 2006 